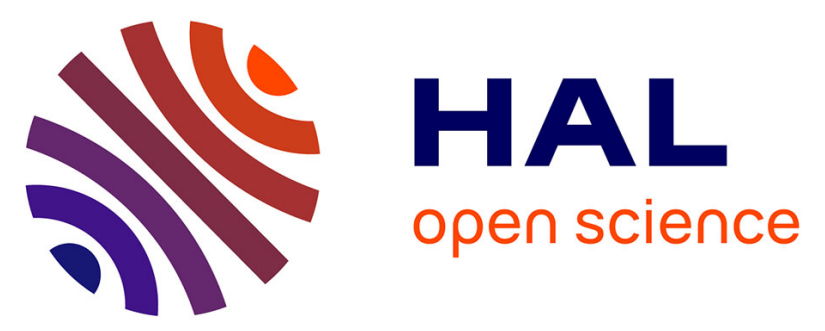

\title{
Étude de l'érosion due au phénomène d'arc dans les contacts de relais des centraux electromécaniques - Corrélation érosion-durée d'arc. Influence de la vapeur d'eau atmosphérique sur la durée d'arc
}

J.L. Queffelec, D. Travers, N. Ben Jemaa, J.P. Treguier, D. Collobert, H. Prigent

\section{To cite this version:}

J.L. Queffelec, D. Travers, N. Ben Jemaa, J.P. Treguier, D. Collobert, et al.. Étude de l'érosion due au phénomène d'arc dans les contacts de relais des centraux electromécaniques - Corrélation érosion-durée d'arc. Influence de la vapeur d'eau atmosphérique sur la durée d'arc. Revue de Physique Appliquée, 1977, 12 (5), pp.885-891. 10.1051/rphysap:01977001205088500 . jpa-00244255

HAL Id: jpa-00244255 https://hal.science/jpa-00244255

Submitted on 1 Jan 1977

HAL is a multi-disciplinary open access archive for the deposit and dissemination of scientific research documents, whether they are published or not. The documents may come from teaching and research institutions in France or abroad, or from public or private research centers.
L'archive ouverte pluridisciplinaire HAL, est destinée au dépôt et à la diffusion de documents scientifiques de niveau recherche, publiés ou non, émanant des établissements d'enseignement et de recherche français ou étrangers, des laboratoires publics ou privés. 
Classification

Physics Abstracts

$6.150-6.515-6.700$

\title{
ÉTUDE DE L'ÉROSION DUE AU PHÉNOMĒNE D'ARC DANS LES CONTACTS DE RELAIS DES CENTRAUX ELECTROMÉCANIQUES
}

\section{Corrélation érosion-durée d'arc. Influence de la vapeur d'eau atmosphérique sur la durée d'arc (*)}

\author{
J. L. QUEFFELEC, D. TRAVERS, et N. BEN JEMAA, \\ Université de Rennes I, Laboratoire de Physique Moléculaire 35031 Rennes Cedex, France. \\ J. P. TREGUier, D. COLlobert et H. PRIGENT \\ C. N. E. T. Département C. P. M./P. M. T. 22301 Lannion, France
}

(Reçu le 20 décembre 1976, révisé le 24 janvier 1977, accepté le 3 février 1977)

\begin{abstract}
Résumé. - Le phénomène d'arc dans les contacts des relais téléphoniques et l'érosion qui en résulte, ont été l'objet d'un grand nombre de travaux. Néanmoins, le problème dépendant de plusieurs paramètres (circuits électriques, atmosphère environnante, paramètres mécaniques, formes de contacts...), les connaissances restent encore partielles. C'est le cas pour les contacts utilisés dans les centraux téléphoniques du type Crossbar. Le but de cette étude est de montrer que l'érosion et la durée d'arc à l'ouverture sont corrélées et d'appliquer les résultats de cette corrélation pour calculer l'érosion sur deux formes différentes de paires de contacts en $\mathrm{AgCu}(90 / 10)$, fonctionnant en atmosphère ambiante $80 \%$ d'humidité relative ou en atmosphère ambiante desséchée $(15 \% \mathrm{~d}$ 'humidité relative).
\end{abstract}

Abstract. - Arcing in electrical contacts of telephone switching relays and subsequent erosion has been the subject of a great deal of work. Nevertheless the problem depends on many parameters (electrical circuits, environmental, mechanical, contact shapes...), of which the knowledge is incomplete. This is the case for the contacts used in Crossbar telephone exchanges. The aim of this work is to show that erosion and arc duration on break are correlated, and to apply the results of this correlation to calculate the erosion of two $\mathrm{Ag} / \mathrm{Cu}(90 / 10)$ contact shapes operating in ambient atmosphere ( $80 \%$ relative humidity) or in ambient dried atmosphere (15\% relative humidity).

1. Introduction. - Les relais sont des composants passifs utilisés en grand nombre, d'une part dans les centraux Crossbar du type Pentaconta et CP 400, dans lesquels ils assurent la commutation et la transmission (relais électromécaniques) et d'autre part dans les nouveaux systèmes électroniques (relais sous ampoules scellées). Les contacts de ces relais doivent assurer la transmission des signaux sans atténuation et ceci pour une durée de vie moyenne initialement prévue correspondant à $4 \times 10^{7}$ opérations. Pour ce faire, les contacts ne doivent pas se détruire (collage, soudage usure...) et les résistances de contact doivent être les plus faibles possible. Or, on observe, en exploitation, une dégradation accélérée dans certains centraux [1] (Baron et coll. 1976) et dans certains circuits. Depuis plusieurs années, il a été observé que cette dégradation est due principalement à deux phénomènes :

(*) Communication présentée au Congrès National de Physique des Plasmas, Paris, 6-10 décembre 1976.
- existence de polluants dans les atmosphères des centraux (poussières, vapeur d'eau, vapeurs organiques, $\left.\mathrm{SO}_{2}, \mathrm{H}_{2} \mathrm{~S}, \mathrm{NO} . ..\right)$ conduisant à la formation de couches ;

- existence de contraintes électriques conduisant au phénomène d'arc.

En première approximation, on attribue aux polluants l'augmentation de la résistance de contact et aux contraintes électriques l'érosion. En réalité, le problème est plus complexe; les deux phénomènes de dégradation ne sont pas dissociables quand les contacts travaillent dans une atmosphère polluée et sous contraintes électriques. Dans certains cas, par exemple, on observe un phénomène d'activation des contacts conduisant à l'augmentation de la durée d'arc (érosion accélérée) ([2] Germer 1951 ; [3] Giay et Pharney1973 ; [4] Shubert 1974 ; [5] Uhrig 1975). De plus, il faut tenir compte des contraintes mécaniques (force de contact, vitesse d'ouverture...) et de la métallurgie du contact (forme, homogénéité, état de surface...). Un grand 
nombre de travaux ont eu trait à ces problèmes au cours de ces dernières années. Ceux-ci ont permis de dégrossir les mécanismes de dégradation et de classer certains matériaux vis-à-vis des défauts pour des conditions opératoires bien définies. En fait, le comportement de chaque type de contact dépend de ses conditions opératoires propres, aussi il est difficile d'extrapoler l'ensemble des résultats connus à ce jour à tous les types de contacts. C'est la raison pour laquelle une action a été décidée par les Télécommunications Françaises pour étudier le comportement des matériaux de contact utilisés dans les systèmes français afin d'améliorer la qualité du service. De telles études ont pour but de classer vis-à-vis des défauts les alliages suivants : $\mathrm{AgCu}$ (90/10), AgAu (90/10), AgPd (70/30)... pour des tensions inférieures à quelques centaines de volts et des courants inférieurs à $2 \mathrm{~A}$ (de telles contraintes pouvant apparaître en régime transitoire), une humidité relative variant de $15 \%$ à $80 \%$ et en présence des polluants caractéristiques des atmosphères industrielles.

L'étude développée ici est axée sur la dégradation due aux arcs, c'est-à-dire principalement l'érosion. Dans un précédent travail [6], nous avons décrit l'ensemble des méthodes, optiques et électriques, qui permettent de caractériser l'arc. Aussi dans une première partie de ce travail, nous donnons les conditions opératoires des contacts et les caractéristiques générales du phénomène de dégradation. Dans une deuxième partie, nous développerons le comportement général de tels contacts à partir d'un cas concret dans le but de corréler l'érosion à la durée moyenne d'arc. Dans une dernière partie, nous étudierons l'influence de la vapeur d'eau dans l'atmosphère ambiante sur la durée d'arc pour deux paires de contacts de formes différentes en $\mathrm{AgCu}$ $(90 / 10)$.

2. Généralités. - 2.1 CoNDITIONS EXPÉRIMENTALES. - Rappelons tout d'abord les conditions dans lesquelles opèrent les relais. Les contacts de relais étudiés sont du type CP 400 ( $\mathrm{AgCu}$ 90/10). Ils sont dissymétriques comme le montre la figure 1 où nous présentons des paires de contacts de géométrie différente. Ces

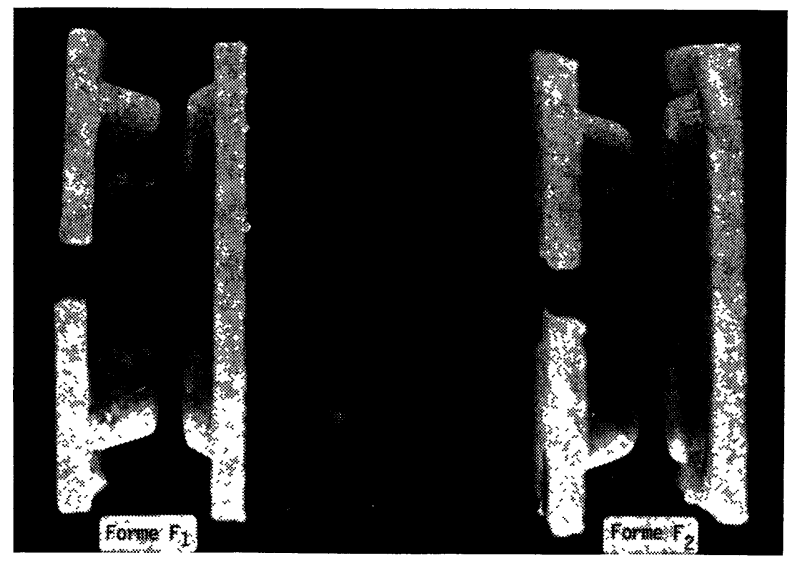

Fig. 1. - Formes des contacts CP 400 utilisés. [Contact shapes of CP 400 used.] contacts sont placés dans un circuit purement résistif pour éviter toute ambiguïté sur les paramètres de l'arc à l'ouverture et à la fermeture, les contraintes électriques étant les suivantes : $v=48 \mathrm{~V}$ et $I$ variable de $200 \mathrm{~mA}$ à $2 \mathrm{~A}$ (Fig. 2). Un oscillateur actionne les contacts à la fréquence de $3 \mathrm{~Hz}$. Dans toute cette étude, le contact le moins volumineux a été choisi comme anode.

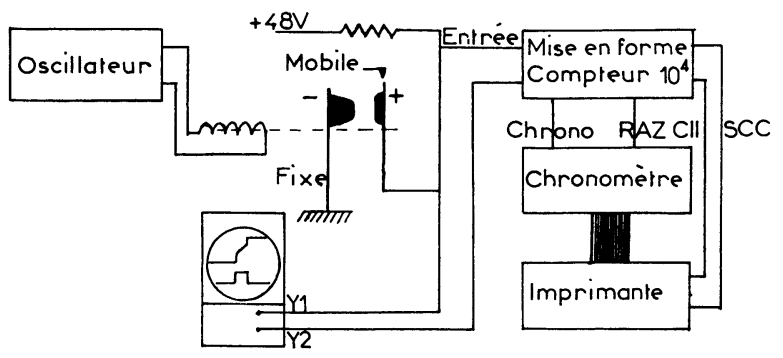

Fig. 2. - Synoptique du montage.

[Schematic of the experiment.]

2.2 Mesure DE LA DURÉE D'ARC. - Dans ce type de circuit, la caractéristique de tension à l'ouverture (Fig. 3) est bien connue ([7] Boddy et coll. 1971 ; [8] Gray 1973) ; elle comprend une phase métallique à

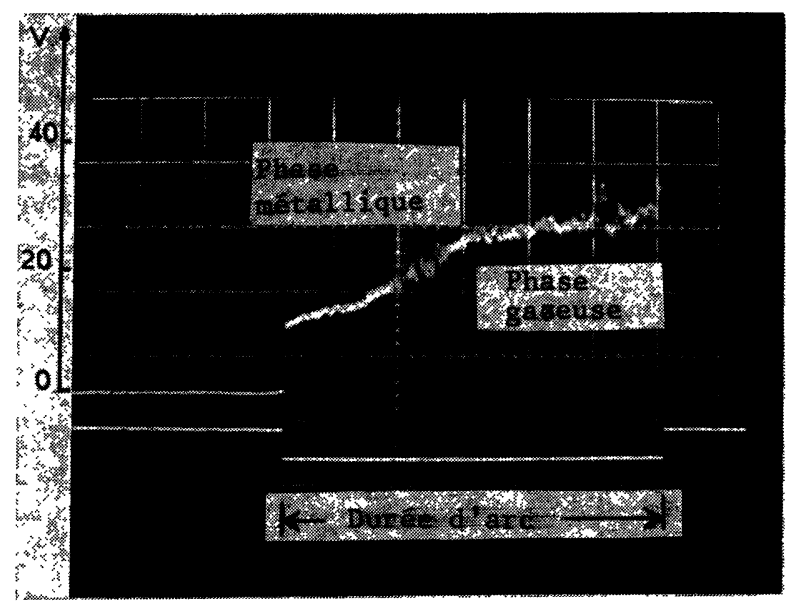

Fig. 3. - Visualisation à l'oscilloscope de la durée d'arc. [Oscilloscope visualization of arc duration.]

environ $12 \mathrm{~V}$ et une phase gazeuse à $25 \mathrm{~V}$. A la fermeture seule une phase métallique existe ; sa durée est très faible par rapport à celle de l'ensemble de deux phases à l'ouverture : nous ne l'avons pas prise en considération dans ce travail.

Le dispositif de mesure de la durée d'arc (Fig. 2) comprend un circuit de mise en forme du signal aux bornes des contacts à l'ouverture (passage de 0 à $48 \mathrm{~V}$ ), un compteur par $10^{n}$, un chronomètre ainsi qu'une imprimante.

Le circuit de mise en forme du signal comprend entre autres, un dispositif à diode Zener qui déclenche deux monostables, le premier sur le front de montée $0-5 \mathrm{~V}$, le second sur le front 40-45 V. La recombinaison de ces 
deux signaux permet d'obtenir une impulsion carrée signal chrono dont la largeur est égale au temps de montée du signal d'entrée : durée des phases métallique et gazeuse, définissant la durée d'arc (Fig. 3).

Le compteur par $10^{n}$ a pour rôle d'ouvrir une porte pendant la durée de $10^{n}$ ouvertures. Il est déclenché par le signal de remise à zéro (R. A. Z.) et bloqué par le signal chrono $10^{n}$. Un fréquencemètre de $1 \mathrm{MHz}$ utilisé en chronomètre totalise les $10^{n}$ durées d'arc à l'ouverture.

Dès le blocage du compteur, une impulsion est envoyée sur l'imprimante : commande d'impression par impulsion (C. I. I.), le nombre affiché sur le chronomètre est alors transcrit sur l'imprimante. A la fin du cycle d'impression, un signal de cycle complémentaire (S. C. C.) est appliqué sur l'entrée R. A.Z. pour déclencher le cycle de comptage suivant.

3. Corrélation érosion. Durée d'arc. -3.1 TeChNIQUES DES MESURES ET MOYENS D'OBSERVATIONS. Cette étude a été réalisée sur des contacts de forme $F_{1}$ (Fig. 1) pour des courants

$$
0,2 \mathrm{~A}<I<1,64 \mathrm{~A},
$$

dans l'atmosphère non contrôlée du laboratoire, susceptible donc de contenir des traces de polluants.

Afin de maintenir constants les paramètres mécaniques, nous avons utilisé la même bobine de relais, et toujours placé les lames contacts qui travaillent à la même position dans le même empilement.

Pour déterminer la variation de masse des contacts (érosion) avec une bonne précision, ceux-ci effectuent d'une manière continue un nombre total d'ouvertures $N$ d'autant plus grand que $I$ est faible. Pour chaque valeur du courant choisi, nous prenons des lames contacts neuves pesées initialement au moyen d'une microbalance. Après les $N$ ouvertures, les contacts sont repesés après lavage à l'alcool (sans grattage) pour les débarrasser d'un dépôt noirâtre très peu adhérent au niveau des contacts (Fig. 4a). Ce dépôt est principalement constitué de carbone amorphe et d'agrégats métalliques [9] (Gray 1976).

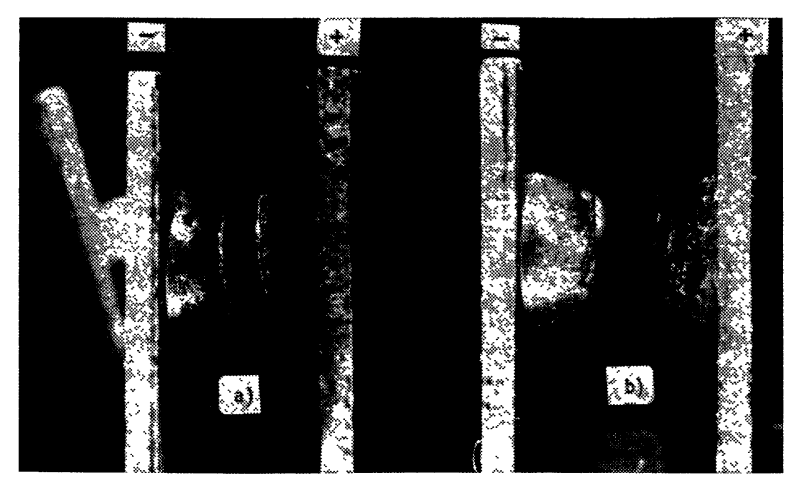

FIG. 4. - Contacts après fonctionnement : a) $I=1,64$ A sans lavage à l'alcool. b) $I=1,44 \mathrm{~A}$ après lavage à l'alcool.

[Contacts after operating. a) $I=1.64$ A without cleaning with alcohol. b) $I=1,44 \mathrm{~A}$ after being cleaned with alcohol.]
Afin de suivre l'évolution de la durée moyenne d'arc, nous avons réglé le compteur par $10^{n}$ sur $10^{4}$ pour des raisons d'appareillage et d'exploitation des résultats.

$\mathrm{Au}$ cours des différentes études, l'évolution de l'aspect des contacts a pu être observée au moyen d'une lunette grossissante, et la caractéristique en tension de l'arc visualisée à l'oscilloscope.

3.2 DURÉE D'ARC ET RÉSULTATS DES OBSERVATIONS. - Les premiers résultats obtenus [6] sur l'étude de l'évolution de la durée moyenne d'arc (sur $10^{4}$ ouvertures) en fonction du nombre d'ouvertures $(N)$ et pour divers courants ont été complétés pour les courants faibles.

Les résultats dans leur ensemble sont donnés sur les figures 5 et 6 . Les observations visuelles à la lunette ainsi qu'à l'oscilloscope nous permettent de faire quelques commentaires sur ces résultats en classant les courants en quatre domaines : forts, moyens, faibles et très faibles.

Courants forts : $I=1,64$ A et $I=1,56$ A.

Au cours du fonctionnement, il y a formation de pics anodiques instables, entraînant une augmentation de la durée moyenne d'arc. Ces pics se détériorent au cours du temps (Fig. 5, points D et E) puis se reforment. Un cratère cathodique apparaît. Nous notons une fluctuation importante de la durée moyenne d'arc par $10^{4}$ ouvertures et cette fluctuation est notablement plus grande d'une ouverture à l'autre. L'arc se produit à chaque ouverture avec phases métallique et gazeuse.

Courants moyens: $I=1,44$ A et $I=0,96$ A.

Pour $I=1,44 \mathrm{~A}$, un pic anodique stable se forme et se maintient jusqu'à l'arrêt du fonctionnement (Fig. 4b).

Pour $I=0,96$ A (Fig. 5), un pic anodique stable se forme progressivement à partir du point $\mathrm{A}$. Son augmentation de volume s'accompagne d'une augmentation de la durée d'arc jusqu'au point $B$ où il y a rupture du pic accompagnée d'une chute brusque de la durée d'arc. Puis en $\mathrm{C}$, un nouveau pic se forme à une cadence plus accélérée, accompagné d'une nouvelle augmentation de la durée d'arc. Comme pour les courants forts la cathode se creuse et l'arc se produit à chaque ouverture avec phases métallique et gazeuse. Par contre, nous notons ici une grande stabilité de la durée moyenne d'arc par tranche de $10^{4}$ ouvertures, ainsi que d'une ouverture à l'autre.

Courants faibles: $I=0,73 \mathrm{~A} ; I=0,55 \mathrm{~A}$ et $I=0,4 \mathrm{~A}$.

Nous n'observons plus de pic anodique. Les fluctuations de la durée moyenne d'arc par tranche de $10^{4}$ ouvertures ainsi que d'une ouverture à l'autre réapparaissent.

Pour $I \leqslant 0,55 \mathrm{~A}$, la phase gazeuse de l'arc disparaît, la phase métallique seule subsiste et sa durée diminue avec $I$. L'arc ne se produit plus à chaque ouverture et ce phénomène s'accentue vers les faibles intensités si bien qu'à $I=0,4 \mathrm{~A}$ environ $50 \%$ des ouvertures 

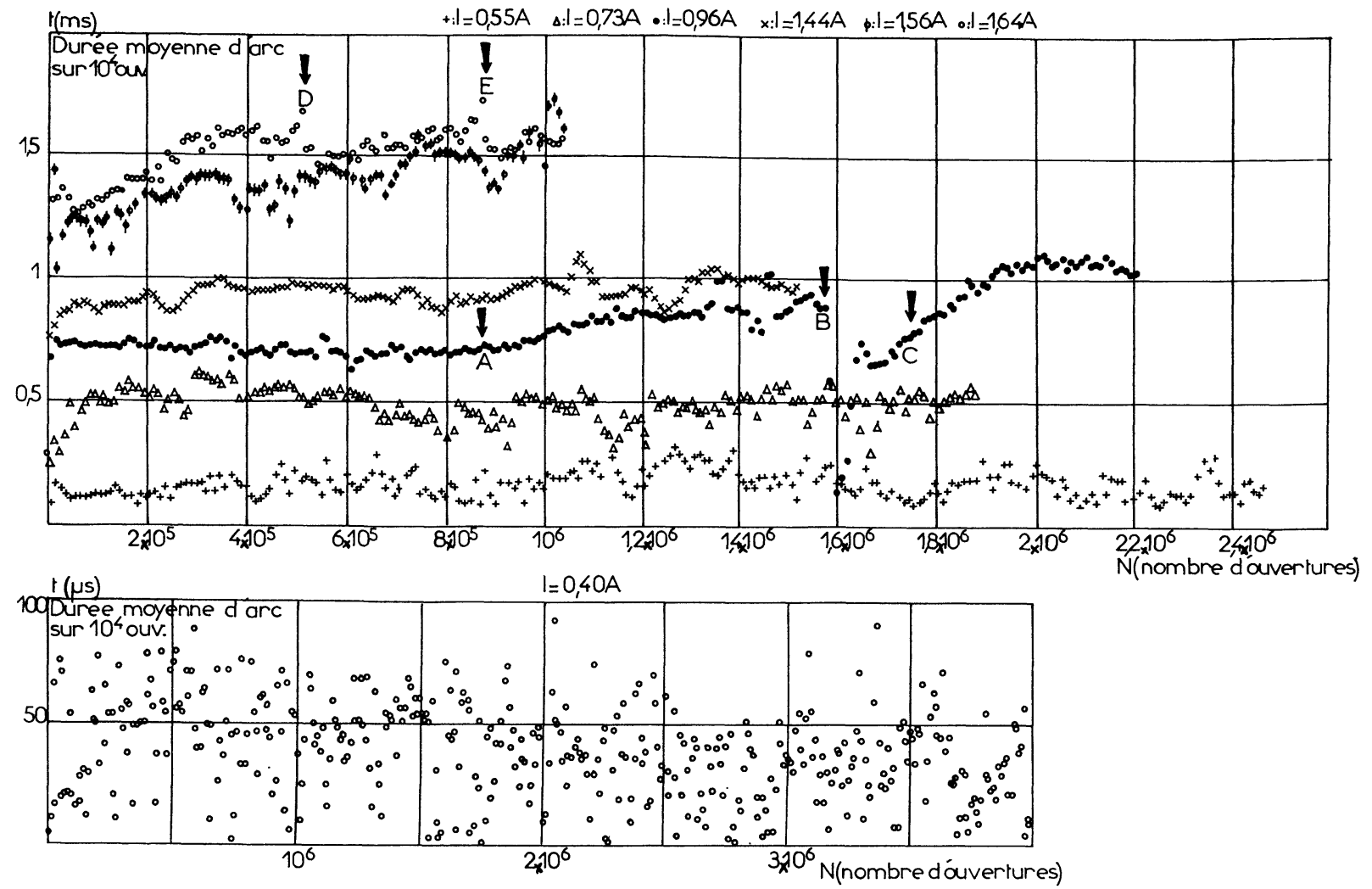

FIg. 5. - Etude en atmosphère ambiante de l'évolution de la durée moyenne d'arc en fonction du nombre d'ouvertures pour différents courants.

[Study in ambient atmosphere of average arc duration as a function of number of operations and current.]

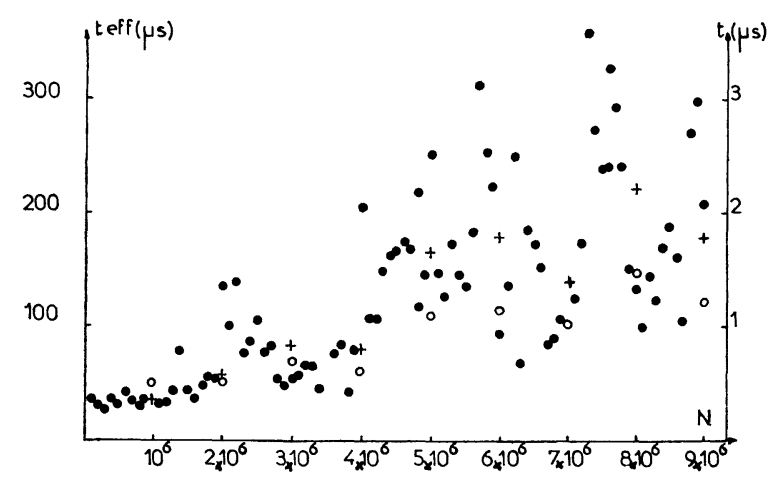

Fig. 6. - Evolution de la durée d'arc pour $I=0,2 \mathrm{~A}$. $\bigcirc$ teff : durée moyenne des arcs effectifs ( $>1 \mu \mathrm{s})$ par tranches de $10^{5}$ ouvertures $;+t_{\text {eff }}$ : durée moyenne des arcs effectifs par tranches de $10^{6}$ ouvertures ; $\bigcirc t$ : durée moyenne générale d'arc par tranches de $10^{6}$ ouvertures.

[Evolution of arc duration for $I=0.2 \mathrm{~A}$. $t_{\mathrm{eff}}$ : average duration of effective arcs ( $>1 \mu \mathrm{s})$ on $10^{5}$ operations. $+t_{\mathrm{eff}}$ : average duration of effective arcs on $10^{6}$ operations. $\bigcirc t$ : average arc duration on $10^{6}$ operations.]

donnent des arcs dont la durée est inférieure à $1 \mu \mathrm{s}$, limite de détection de notre chronomètre.

Courants très faibles : $I=0,2$ A (Fig. 6).

Pour ce courant un dispositif supplémentaire de comptage a été mis en place afin de déterminer le nombre d'arcs par tranches de $10^{4}$ ouvertures pour lesquels la durée est supérieure à $1 \mu$ s (arcs effectifs). Ceci nous a permis de déterminer la probabilité d'obtention d'un arc effectif à l'ouverture ; celle-ci passe de $1,4 \%$ au début du fonctionnement à $0,7 \%$ après $9 \times 10^{6}$ ouvertures. Ceci est conforme aux résultats de [10] (Holm 1967) relatifs à une valeur minimum de courant d'arc $(I=0,4 \mathrm{~A})$.

3.3 Erosion. - Les résultats sont donnés par le tableau de la figure 7 et les courbes de la figure 8 . Nous constatons que dans ce type de contacts l'érosion de la cathode se traduit par une perte de masse pour toutes les valeurs de courants utilisés. Par contre, l'anode ne subit de perte de masse (érosion) que pour des courants $I<0,8 \mathrm{~A}$, au-delà de cette limite, il y a un transfert de masse de la cathode vers l'anode.

Nous avons calculé à partir des résultats de la figure 7 que l'érosion cathodique est proportionnelle à $I^{4}$ pour des courants inférieurs à $1 \mathrm{~A}$ (domaine principal des télécommunications) tandis que la durée d'arc est proportionnelle à $I^{3}$ [6]. La courbe de la figure 9 donne la corrélation entre la perte de masse de la cathode et la durée moyenne d'arc ; ainsi cette courbe permet de déterminer l'érosion cathodique à partir de la détermination expérimentale de la durée moyenne d'arc : donc de prévoir la durée de vie des contacts. 
ÉROSION DES CONTACTS DE RELAIS TÉLÉPHONIQUES

\begin{tabular}{|c|c|c|c|c|c|c|c|c|c|c|}
\hline$(A)$ & 0,20 & 0,40 & 0,55 & 0,73 & 0,96 & 1,44 & 1,56 & 1,64 & 1,15 & 1,30 \\
\hline$N \times 10^{-4}$ & 900 & 404 & 247 & 188 & 221 & 152 & 104 & 104 & 20 & 20 \\
\hline$t(\mathrm{~ms})$ & 0,0009 & 0,039 & 0,173 & 0,492 & 0,811 & 0,941 & 1,40 & 1,51 & 0,860 & 0,910 \\
\hline$\Delta M_{\mathrm{c}} \times 10^{10}(\mathrm{~g})$ & $-0,088$ & $-0,21$ & $-1,96$ & $-4,60$ & $-16,2$ & $-26,2$ & $-40,4$ & & & \\
\hline$\Delta M_{\mathrm{a}} \times 10^{10}(\mathrm{~g})$ & $-0,130$ & $-0,18$ & $-0,70$ & $-1,04$ & $+5,09$ & $+17,9$ & $+22,8$ & & & \\
\hline$W_{\mathrm{c}} \times 10^{6}\left(\mathrm{gc}^{-1}\right)$ & & & $-2,05$ & $-1,28$ & $-2,08$ & $-1,93$ & $-1,84$ & & & \\
\hline
\end{tabular}

FIG. 7. - Durée moyenne d'arc et variation de masse des contacts $: N:$ nombre total d'ouvertures. $t:$ durée moyenne d'arc par ouverture. $\Delta M_{\mathrm{C}}$ : variation de masse de la cathode par ouverture. $\Delta M_{\mathrm{A}}:$ variation de masse de l'anode par ouverture. $W_{\mathrm{c}}:$ variation de masse de la cathode par Coulomb.

[Average arc duration and contact weight variations. $N$ : total number of operations. $t:$ average arc duration on break. $\Delta M_{\mathrm{C}}$ : cathode weight variations per operation. $\Delta M_{\mathrm{A}}$ : anode weight variation per operation. $W_{\mathrm{c}}$ : cathode weight variation per Coulomb.

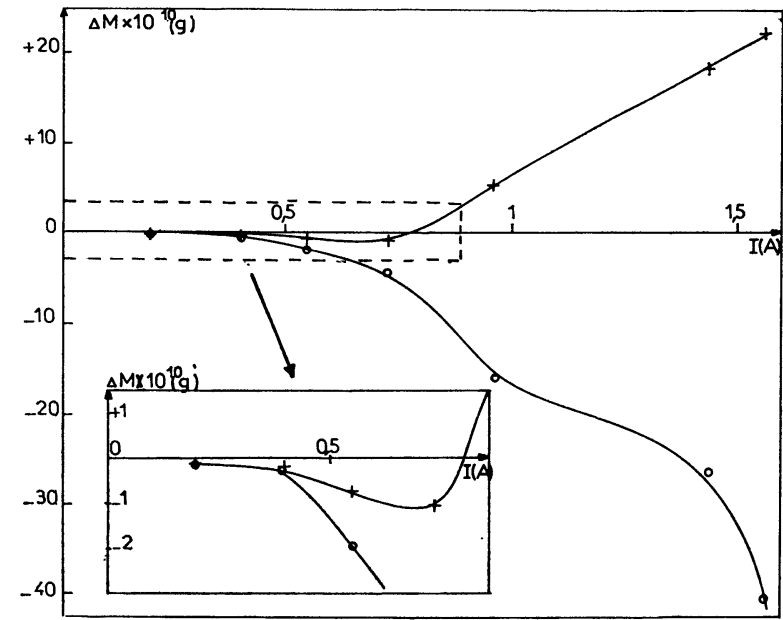

Fig. 8. - Variation de masse des contacts par ouverture en fonction du courant. $O$ : cathode; + anode.

[Contact weight variations as function of current. $\mathrm{O}:$ cathode ; + : anode.

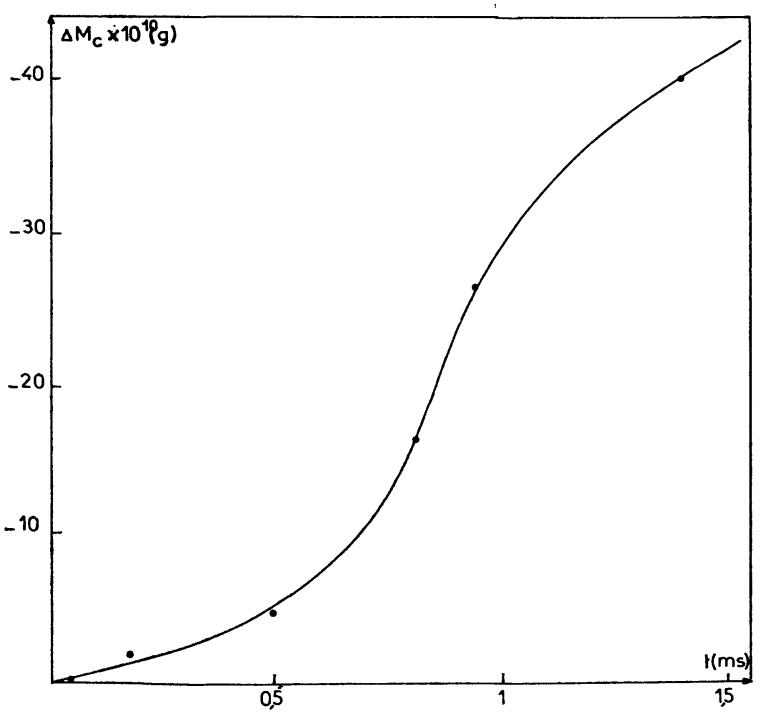

Fig. 9. - Variation de masse de la cathode par ouverture en fonction de la durée moyenne d'arc.

[Cathode weight variations per operation as a function of average arc duration.
3.3.1 Précision des résultats. - La précision des appareils de mesure donnant la variation de masse cathodique ainsi que la durée moyenne d'arc est telle que ces résultats sont donnés à $12 \%$ près dans les cas les plus défavorables $(I<0,4 \mathrm{~A})$. Cette précision s'améliore avec l'augmentation de $I$ et atteint $1 \%$ à partir de $I=1 \mathrm{~A}$.

Une seule mesure d'érosion cathodique et de durée moyenne d'arc a été faite pour chaque courant. Cependant en raison du nombre important d'intensités de courant utilisé, nous estimons que les marges d'erreur sur les points expérimentaux des figures 8 et 9 sont faibles.

\section{Influence de la vapeur d'eau atmosphérique sur} la durée d'arc. - Le mécanisme de l'activation des contacts par les polluants organiques a été étudié par Gray et coll. [11,12] : les vapeurs organiques adsorbées par la surface des contacts arrivent progressivement dans l'arc où elles se décomposent avec production de particules carboniques qui se déposent sous forme de poudre noire sur les contacts. Gray et coll. [13] citent que Haworth a montré que dans une atmosphère à $100 \%$ d'humidité relative, l'activation pour des contacts en $\mathrm{Pd}$ pur est complètement supprimée : la poudre noire caractérisant généralement l'activation des contacts disparaît totalement. Cet auteur considère que la vapeur d'eau adsorbée en couche mince sur les contacts protège ceux-ci contre les polluants donc de l'activation.

Dans le but d'étudier cette influence de la vapeur d'eau atmosphérique sur les contacts $\mathrm{Ag} / \mathrm{Cu}(90 / 10)$ nous avons mis au point un dispositif à deux voies (Fig. 10) permettant l'étude simultanée de durée d'arc de deux paires de contacts fonctionnant dans les mêmes conditions mécaniques et électriques: l'une dans l'atmosphère ambiante du laboratoire où le degré d'humidité relative est sensiblement de $80 \%$ et l'autre dans une enceinte renfermant cette même atmosphère desséchée par du tamis moléculaire (humidité relative $15 \%$ ).

Cette étude a été faite avec des paires de contacts de 


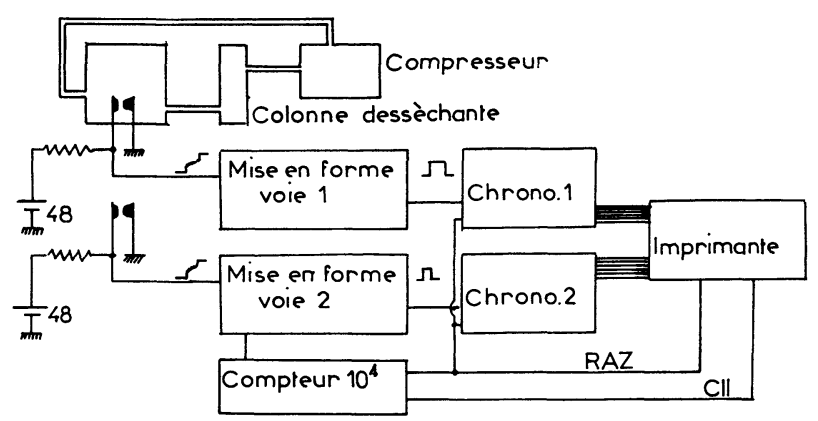

Fig. 10. - Schéma de l'ensemble du montage pour l'étude comparative des durées d'arcs en atmosphère sèche $(15 \%$ d'humidité relative) et en atmosphère ambiante (80\% d'humidité relative).

[General scheme of experiment for comparative studies of arc duration in dry atmosphere (15\% relative humidity) and in ambient atmosphere ( $80 \%$ relative humidity).]

formes $F_{1}$ et $F_{2}$ et les résultats sont donnés par la figure 11 et le tableau de la figure 12 .

D'une manière générale, nous constatons que les durées moyennes d'arc dans l'atmosphère sèche sont supérieures à celles obtenues dans l'atmosphère
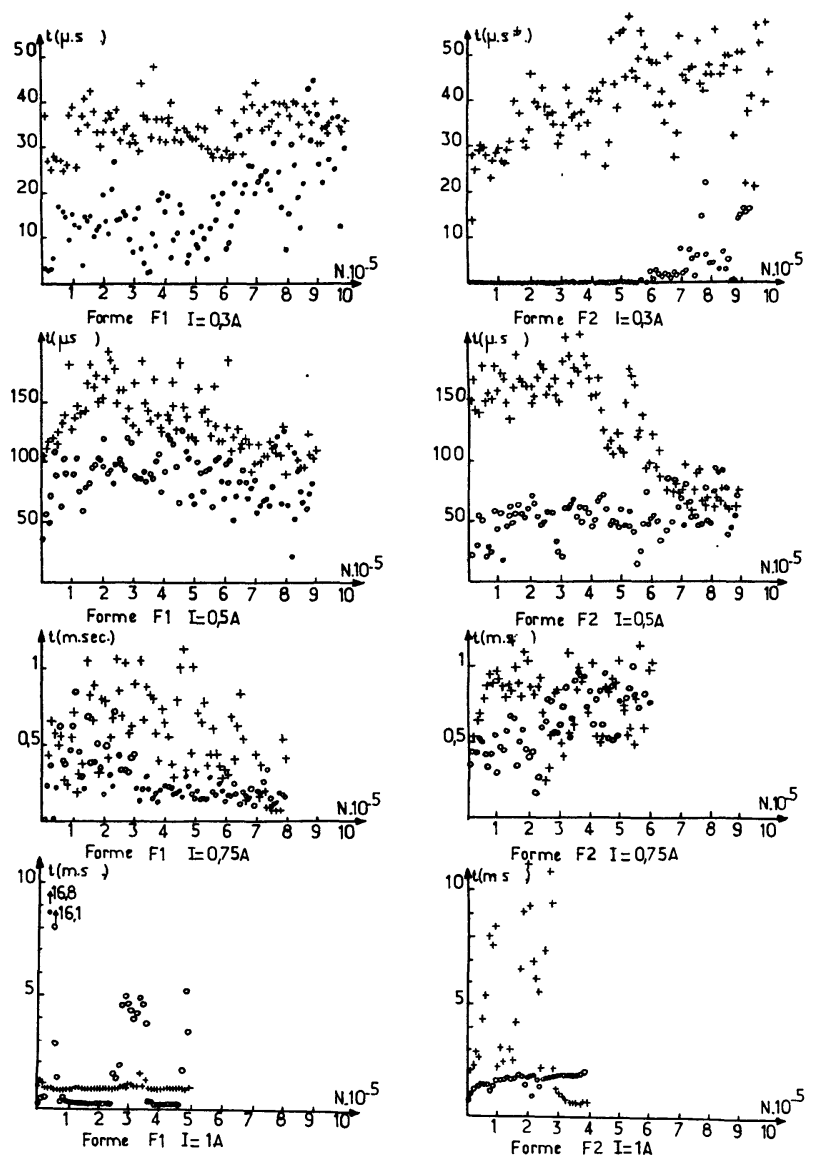

Fig. 11. - Evolution de la durée d'arc en fonction du nombre d'ouvertures et du degré d'humidité de l'atmosphère. $+: 15 \%$ d'humidité relative. $\mathrm{O}: 80 \%$ d'humidité relative.

[Evolution of arc duration as function of operating number and atmosphere humidity. $+: 15 \%$ relative humidity. $O: 80 \%$ relative humidity.]
$I(A)$

$\begin{array}{rrrrr} & 0,3 & 0,5 & 0,75 & 1 \\ t_{\mathrm{S}}(\mu \mathrm{s}) & 34 & 132 & 508 & 943\end{array}$

Forme $F_{1}$

$\begin{array}{lrrrr}t_{\mathrm{A}}(\mu \mathrm{s}) & 17 & 87 & 263 & 2060 \\ t_{\mathrm{S}}(\mu \mathrm{s}) & 40 & 130 & 781 & 3990\end{array}$

Forme $\mathrm{F}_{2}$

$\begin{array}{lllll}t_{\mathrm{A}}(\mu \mathrm{s}) & 5 & 52 & 639 & 1597\end{array}$

Fig. 12. - Tableau des résultats pour les deux formes de contacts $F_{1}$ et $F_{2}$ et pour les deux degrés d'humidité de l'atmosphère : $t_{\mathrm{S}}$ : durée moyenne d'arc à $15 \%$ d'humidité relative.

$t_{\mathrm{A}}:$ durée moyenne d'arc à $80 \%$ d'humidité relative.

[Table of results for the two contact shapes $F_{1}$ and $F_{2}$ and for two degrees of relative atmosphere humidity. $t_{\mathrm{S}}$ : average arc duration at $15 \%$ relative humidity. $t_{\mathrm{A}}$ : average arc duration at $80 \%$ relative humidity.]

ambiante. Leur rapport varie de 1,5 à 2,5 environ pour les deux formes.

Des mesures d'érosion ont été faites pour $I=1 \mathrm{~A}$ avec la forme $F_{2}$. Les résultats donnent :

$$
\frac{t_{\mathrm{S}}}{t_{\mathrm{A}}}=2,49 \text { et } \frac{\Delta M_{\mathrm{c}} \text { (At. sèche) }}{\Delta M_{\mathrm{c}} \text { (At. ambiante) }}=3,17 .
$$

Ceci montre que l'érosion cathodique est augmentée sensiblement dans le même rapport que les durées moyennes d'arc.

Les résultats de la durée moyenne d'arc(Figs. 11 et 12) appellent certaines remarques :

- Toute condition égale par ailleurs mise à part, la forme des contacts, l'ordre de grandeur des durées d'arc sont comparables.

- Les durées moyennes d'arc dans l'atmosphère ambiante pour la forme $F_{1}$ sont sensiblement identiques à celles obtenues dans l'étude précédente $(\S 3)$.

- Pour la forme $\mathrm{F}_{1}$ à $I=1 \mathrm{~A}$ (Fig. 11) le rapport $t_{\mathrm{S}} / t_{\mathrm{A}}$ est inférieur à 1 . Ceci est dû à un certain nombre de points aberrants pouvant provenir d'une activation momentanée des contacts.

- Dans presque tous les cas, le rapport $t_{\mathrm{S}} / t_{\mathrm{A}}$ tend vers 1 après un nombre plus ou moins grand d'ouvertures. Nous n'expliquons pas pour le moment ce phénomène.

- Pour les courants faibles $(I<$ courant minimum d'arc), on a constaté une probabilité d'obtention d'arc importante $(90 \%)$ en atmosphère sèche alors qu'elle est seulement de 5 à $10 \%$ environ dans l'atmosphère ambiante. La réduction du degré d'humidité dans notre atmosphère ambiante en abaissant le courant minimum d'arc [10] augmente la probabilité d'obtenir un arc.

4. Conclusion. - Dans cette étude sur les contacts de relais CP $400 \mathrm{AgCu}$ (90/10), nous avons montré qu'il existe une corrélation entre la durée moyenne 
d'arc à l'ouver ture et la variation de masse des contacts fonctionnant dans l'atmosphère ambiante. Cette corrélation, établie de manière quantitative permet donc, par simple mesure de la durée moyenne d'arc de prévoir l'érosion des contacts et ainsi de déterminer leur durée de vie. L'étude comparative de l'érosion des contacts dans différentes atmosphères contrôlées, se ramène donc à la mesure des durées moyennes d'arc correspondantes.

L'application de cette méthode, dans le cas où l'on agit sur le paramètre degré d'humidité de l'atmosphère, nous a permis de montrer que l'érosion est multipliée par un facteur 1,5 à 2,5 quand on passe de l'atmosphère ambiante ( $80 \%$ d'humidité relative) à cette même atmosphère, mais desséchée (15\% d'humidité relative), et ceci pour les deux formes $F_{1}$ et $F_{2}$.

Ces techniques expérimentales doivent nous permettre, non seulement de classer, vis-à-vis de l'érosion, les différents alliages constituant les contacts des relais électromécaniques ( $\mathrm{AgCu}, \mathrm{AgAu}, \mathrm{AgPd} . .$.$) , mais aussi$ d'étudier des relais sous ampoules scellées dans les nouveaux systèmes.

\section{Bibliographie}

[1] Baron, J. P., Le Traon, J. Y., Riou, M. T. et Auregan, M., Holm Seminar on electrical Contacts, Chicago, sept. 1976.

[2] Germer, L. H., J. Appl. Phys. 22 (1951) 955-964.

[3] Gray, E. W. et Pharney, J. R., J. Appl. Phys. 44 (1973), 2124-2129.

[4] SChubert, R., J. Appl. Phys. 45 (1974) 2422-2427.

[5] Uhrig, T. A., J. Appl. Phys. 46 (1975) 4705-4711.

[6] Treguier, J. P., Ben JemaA, N., Collobert, D., Prigent, H. et QuefFeleC, J. L., Holm Seminar on electrical contacts Chicago, sept. 1976.

[7] Boddy, P. J. et Utsumi, T., J. Appl. Phys. 42 (1971), 33693373.
[8] Gray, E. W., I. E. E. E., Trans. Plasma Sci. PS 1 (1973) 1, 30-33.

[9] Gray, E. W., I. E. E. E. Trans. Parts Hybrids and Packaging PHP 12 (1976) 1, 11-15.

[10] Holm, R., Electric Contacts, (Springer, New York) 1967, 4th ed., 274-286.

[11] Gray, E. W. et MCKNIGHT, L. G., Int. J. Electronics 37 (1974) $257-260$

[12] Gray, E. W. et McKnight, L. G., I. E. E. E., Trans Parts Hybride and Packaging PHP 11 (1975) 2, 121-124.

[13] Gray, E. W. et MCKNIGHT, L. G., Int. J. of Mass Spectrometry and Ion Physics 17 (1975) 353-370. 